\title{
A novel approach to non-commutative gauge theory
}

\author{
Vladislav G. Kupriyanov ${ }^{a, b, 1}$ and Patrizia Vitale ${ }^{c, d}$ \\ ${ }^{a} C M C C$, Universidade Federal do $A B C$, \\ 09210-580 Santo André, SP, Brazil \\ ${ }^{b}$ Physics Department, Tomsk State University, \\ Tomsk 634050, Russia \\ ${ }^{c}$ Dipartimento di Fisica "E. Pancini", Università di Napoli Federico II, \\ Complesso Universitario di Monte S. Angelo Edificio 6, via Cintia, 80126 Napoli, Italy \\ ${ }^{d}$ INFN - Sezione di Napoli, \\ Complesso Universitario di Monte S. Angelo Edificio 6, via Cintia, 80126 Napoli, Italy \\ E-mail: vladislav.kupriyanov@gmail.com, patrizia.vitale@na.infn.it
}

ABSTRACT: We propose a field theoretical model defined on non-commutative space-time with non-constant non-commutativity parameter $\Theta(x)$, which satisfies two main requirements: it is gauge invariant and reproduces in the commutative limit, $\Theta \rightarrow 0$, the standard $\mathrm{U}(1)$ gauge theory. We work in the slowly varying field approximation where higher derivatives terms in the star commutator are neglected and the latter is approximated by the Poisson bracket, $-i[f, g]_{\star} \approx\{f, g\}$. We derive an explicit expression for both the NC deformation of Abelian gauge transformations which close the algebra $\left[\delta_{f}, \delta_{g}\right] A=\delta_{\{f, g\}} A$, and the NC field strength $\mathcal{F}$, covariant under these transformations, $\delta_{f} \mathcal{F}=\{\mathcal{F}, f\}$. NC ChernSimons equations are equivalent to the requirement that the NC field strength, $\mathcal{F}$, should vanish identically. Such equations are non-Lagrangian. The NC deformation of Yang-Mills theory is obtained from the gauge invariant action, $S=\int \mathcal{F}^{2}$. As guiding example, the case of $s u(2)$-like non-commutativity, corresponding to rotationally invariant NC space, is worked out in detail.

Keywords: Gauge Symmetry, Non-Commutative Geometry

ArXiv ePrint: 2004.14901

\footnotetext{
${ }^{1}$ ORCID:0000-0003-0105-8418.
} 


\section{Contents}

1 Introduction 1

2 Non-commutative U(1) gauge algebra 3

3 Non-commutative field strength $\quad 6$

4 Noncommutative Chern-Simons model 9

5 Non-commutative Yang-Mills theory 10

6 Conclusions 11

$\begin{array}{ll}\text { A Gauge connection } & 11\end{array}$

\section{Introduction}

Noncommutative gauge theories have been widely studied in the past years mixing successes and defeats. It is of common knowledge that, while space-time noncommutativity represents a natural resolution of the clash between general relativity and quantum mechanics in strong gravitational fields (see for example [1]), it doesn't give rise to well defined quantum field theories, which are generically affected by the so called UV/IR mixing [2,3], except for a few models with very special features. It is out of the scope of this paper to present a comprehensive review of the subject. We shall just focus on the aspects which shall be addressed here.

For the purposes of the paper, the "classical picture" which we refer to, is that of a noncommutative theory of gauge and (when included) matter fields, which is described in terms a noncommutative algebra $(\mathcal{A}, \star)$ representing space-time, a right $\mathcal{A}$-module, $\mathbb{M}$, representing matter fields, a group of unitary automorphisms of $\mathbb{M}$ acting on fields from the left, representing $\mathrm{U}(N)$ gauge transformations. ${ }^{1}$ In such a framework, the dynamics of fields is described by means of a natural differential calculus based on derivations of the NC algebra [4-9]. Moreover, the gauge connection is the standard noncommutative analog of the Koszul notion of connection [8-11]. (See appendix A for a brief review of the latter approach. For a physically inspired perspective see the pioneering work [12].)

Therefore, it is evident that, in the classical framework, one major problem is to have a well defined differential calculus, namely, an algebra of $\star$-derivations of $\mathcal{A}$ such that

$$
D_{a}(f \star g)=D_{a} f \star g+f \star D_{a} g .
$$

\footnotetext{
${ }^{1}$ Notice however that in the paper we shall only consider pure gauge theories.
} 
For constant noncommutativity, assuming $\Theta$ to be non-degenerate, the latter are successfully realised by star commutators

$$
D_{a} f=\left(\Theta^{-1}\right)_{a b}\left[x^{b}, f\right]_{\star} \stackrel{\Theta \rightarrow 0}{\longrightarrow} \partial_{a} f
$$

thus reproducing the correct commutative limit. For coordinate dependent $\Theta(x)$ the situation is much more complicated. Lie algebra type star products,

$$
\left[x^{j}, x^{k}\right]_{\star}=c_{l}^{j k} x^{l}
$$

do admit a generalisation of (1.2) according to

$$
D_{j} f=k\left[x^{j}, f\right]_{\star}
$$

with $k$ a suitable dimensionful constant, but the limit, $\Theta \rightarrow 0$, does not yield the correct commutative limit (see [13-17] for details and applications). A related approach, is to use twisted differential calculus for those NC algebras whose star product is defined in terms of a twist [18-22].

To summarise, for generic coordinate dependence of $\Theta$, where one needs to employ the general Kontsevich star product [23],

$$
f \star g=f \cdot g+\frac{i}{2} \Theta^{a b}(x) \partial_{a} f \partial_{b} g+\ldots,
$$

ordinary derivations violate Leibniz rule,

$$
\partial_{c}(f \star g)=\left(\partial_{c} f\right) \star g+f \star\left(\partial_{c} g\right)+\frac{i}{2} \partial_{c} \Theta^{a b}(x) \partial_{a} f \partial_{b} g+\ldots
$$

whereas twisted or star derivations, although giving rise to a well defined differential calculus, might not reproduce the correct commutative limit. The problem is not new and several attempts to its solution can be found in the literature.

Other than identifying a differential calculus which be compatible with noncommutativity and yield back the correct commutative limit, we mentioned another problem which emerges in NCQFT, that is the UV/IR mixing, which certainly affects QFT with constant noncommutativity and may or may not affect coordinate dependent cases. Given the important role that NC field theory may play as an effective field theory implementing quantum gravity effects in some low energy regime [1], it is therefore worth to explore a novel approach which might help overcoming some of the problems encountered so far.

Here we shall consider specifically pure gauge theories (no matter fields) and address the problem from a different perspective. Namely, we shall investigate how to modify the very definition of gauge fields and gauge transformations in such a way that they be compatible with space-time noncommutativity and reproduce the correct commutative limit. We propose a novel strategy, which is inspired by a recent approach to gauge theories $[24,25]$, which is based on the conjecture that any well defined gauge theory, including noncommutative and non-associative ones, can be consistently constructed by bootstrapping some starting commutative gauge theory with a noncommutative (resp. non-associative) deformation in such a way to complete some $L_{\infty}$ algebra (see [26] for a physically oriented review 
of the role of $L_{\infty}$ algebras in field theory). However the purpose of the paper is to show that it is possible to follow a simpler, constructive approach, which can be autonomously understood, without recurring to the technical complexity of dealing with $L_{\infty}$ algebras. Before proceeding further, an important remark is in order. Although the procedure is well defined for general space-time non-commutativity, we shall work within a simplified scheme, which amounts to replace $\star$ commutators with Poisson brackets. Then, in order for the construction to be consistent, as long as the product is considered, in eq. (1.5) only the zeroth order in the deformation parameter has to be retained. Strictly speaking this means that space-time stays commutative, but it becomes a Poisson manifold with nontrivial Poisson bracket among position coordinates. Gauge parameters in turn, which are space-time functions, inherit such a non-trivial Poisson structure. It is therefore natural to require that they close under Poisson brackets and it is a legitimate question to ask how gauge theories have to be modified in order to preserve gauge covariance. We shall see that there is no conceptual issue in generalising the whole construction to a genuine non-commutative spacetime, although computationally more complicated.

The paper is organised as follows. In section 2 the Poisson algebra of deformed gauge transformations is introduced and two guiding examples are described, respectively with constant and Lie algebra type noncommutativity. In section 3 a recursive equation for the field strength definition is established, and solved order by order in the deformation parameter. Sections 4 and 5 contain respectively applications to Chern-Simons and YangMills theories. In section 6 we summarise our findings and discuss future perspectives. Finally, appendix A contains a short review of gauge connections and field strength in derivation-based NC gauge theories.

\section{Non-commutative $\mathrm{U}(1)$ gauge algebra}

Let us consider noncommutative space-time represented by the algebra $\mathcal{A}_{\Theta}$ with nonconstant non-commutativity parameter $\Theta(x) .^{2}$ We look for a deformed theory of gauge fields which satisfies two main requirements: it is gauge invariant and reproduces in the commutative limit, $\Theta \rightarrow 0$, the standard gauge theory.

For conventional $\mathrm{U}(1)$ gauge transformations, $\delta_{f}^{0} A=\partial f$, gauge parameters close un Abelian algebra, $\left[\delta_{f}^{0}, \delta_{g}^{0}\right]=0$. For non-Abelian gauge theories where gauge parameters are valued in a non-Abelian Lie algebra, $\mathbf{f}=f_{i} \tau^{i}$, we have instead $\delta_{\mathbf{f}}^{0} A=\partial \mathbf{f}-i[A, \mathbf{f}]$ so that

$$
\left[\delta_{\mathbf{f}}^{0}, \delta_{\mathbf{g}}^{0}\right] A=\partial[\mathbf{f}, \mathbf{g}]-i[A,[\mathbf{f}, \mathbf{g}]]=\delta_{[\mathbf{f}, \mathbf{g}]}^{0} A .
$$

Namely, the algebra of gauge parameters closes with respect to a non-Abelian Lie bracket. Noncommutative $\mathrm{U}(1)$ gauge theory, with gauge parameters now belonging to $\mathcal{A}_{\Theta}$ behaves very much like non-Abelian theories in many respects. Therefore we shall require that the algebra of gauge parameters closes with respect to the star commutator, namely

$$
\left[\delta_{f}, \delta_{g}\right] A=\delta_{-i[f, g]_{\star}} A .
$$

\footnotetext{
${ }^{2}$ We use the symbol capital $\Theta$ for the NC tensor, $\left[x^{i}, x^{j}\right]=\Theta^{i j}(x)$, and lowercase $\theta$ to indicate a small, real parameter.
} 
However, if gauge connections are defined as in appendix A, with gauge transformation

$$
A^{\prime}=A+\partial f-i[A, f]_{\star}
$$

by composing two such transformations we get the result (2.1) only if $\partial$ is a derivation of the star commutator, which, as we have discussed in previous section, in general is not the case.

Our aim in this section is somehow dual to what is usually done, namely, instead of looking for a deformed differential calculus, we shall deform the very definition of gauge transformations,

$$
\delta_{f}^{0} A \rightarrow \delta_{f} A=\partial f+\ldots
$$

in such a way that (2.1) be satisfied.

As already noted, we shall work in the slowly varying, but not necessarily small fields. In such a case we discard higher derivatives terms in the star commutator and take,

$$
-i[f, g]_{\star} \approx\{f, g\}=\Theta^{a b}(x) \partial_{a} f \partial_{b} g .
$$

In the approximation which we have chosen eq. (2.1) becomes

$$
\left[\delta_{f}, \delta_{g}\right] A=\delta_{\{f, g\}} A .
$$

and we look for gauge transformations in the form (2.3) which be compatible with the latter.

A remark is here in order. In the chosen approximation, space-time is still commutative, namely the product between fields is the usual point-wise product, but its geometry is deformed, because it acquires a non-trivial Poisson bracket. Therefore one should rather talk about commutative field theory on Poisson-deformed space-time. Once such a distinction made, in the following we shall refer to the latter as noncommutative space time without any further specification, unless otherwise stated.

A solution to this problem has been proposed in [24, 27] in terms of field dependent gauge transformations, in the form

$$
\delta_{f} A_{a}=\gamma_{a}^{k}(A) \partial_{k} f+\left\{A_{a}, f\right\},
$$

Indeed, it may be verified that the latter close the algebra (2.5) if the matrix $\gamma(A)_{a}^{k}$ satisfies the equation, ${ }^{3}$

$$
\gamma_{b}^{l} \partial_{A}^{b} \gamma_{a}^{k}-\gamma_{b}^{k} \partial_{A}^{b} \gamma_{a}^{l}+\Theta^{l m} \partial_{m} \gamma_{a}^{k}-\Theta^{k m} \partial_{m} \gamma_{a}^{l}-\gamma_{a}^{m} \partial_{m} \Theta^{l k}=0,
$$

where we set, $\gamma_{a}^{k(0)}=\delta_{a}^{k}$, to ensure the correct commutative limit. For arbitrary noncommutativity parameter $\Theta^{k l}(x)$ eq. (2.7) was solved in the form of a perturbative series [27],

$$
\begin{aligned}
\gamma_{a}^{k}(A)=\sum_{n=0}^{\infty} \gamma_{a}^{k(n)}= & \delta_{a}^{k}-\frac{1}{2} \partial_{a} \Theta^{k b} A_{b} \\
& -\frac{1}{12}\left(2 \Theta^{c m} \partial_{a} \partial_{m} \Theta^{b k}+\partial_{a} \Theta^{b m} \partial_{m} \Theta^{k c}\right) A_{b} A_{c}+\mathcal{O}\left(\Theta^{3}\right)
\end{aligned}
$$

\footnotetext{
${ }^{3}$ The convention used here is: the partial derivative with the upper index is the derivation with respect to the field, $\partial_{A}^{b}=\partial / \partial A_{b}$, while the partial derivative with the lower index is a derivation with respect to coordinate, $\partial_{m}=\partial / \partial x^{m}$.
} 
Note that the order of each term $\gamma_{a}^{k(n)}$ in the gauge fields $A$ coincides with the order of this term in the deformation parameter $\Theta$. We also stress here that the Ansatz in eq. (2.6) takes into account only the leading order contribution in derivatives $\partial f$ and $\partial A$. However all orders in $\Theta$ are included, this being necessary to close the algebra (2.5). In this sense eq. (2.6) is exact.

For some specific choices of non-commutativity, one may also discuss the convergence of the series (2.8) and exhibit a closed expression for the gauge transformation (2.6). Here we discuss two particular cases.

Canonical non-commutativity. Canonical non-commutativity corresponds to constant NC parameter $\Theta^{k l}$. Since, $\partial_{m} \Theta^{k l}=0$, the constant solution $\gamma_{a}^{k}=\delta_{a}^{k}$ solves eq. (2.7), yielding the gauge transformations,

$$
\delta_{f} A_{a}=\partial_{a} f+\left\{A_{a}, f\right\} .
$$

Let us notice here that $\gamma_{a}^{k}=\delta_{a}^{k}$ is also a solution for the fully noncommutative case where we replace Poisson brackets by $\star$-commutators. Eq. (2.9) becomes $\delta_{f} A_{a}=\partial_{a} f-i\left[A_{a}, f\right]_{\star}$., which coincides with the standard definition of $\mathrm{NC}$ gauge transformation (2.2) and eq. (2.1) is satisfied.

Lie algebra noncommutativity: $\mathbb{R}_{\theta}^{3}$. The three dimensional rotationally invariant non-commutative space, $\mathbb{R}_{\theta}^{3}$, [13, 14, 28-32] corresponds in the approximation we have chosen, to the $s u(2)$-like Poisson algebra,

$$
\left\{x^{k}, x^{l}\right\}=2 \theta \varepsilon^{k l}{ }_{m} x^{m},
$$

where the real number $\theta$ is a small parameter and $\varepsilon^{k l m}$ is the Levi-Civita symbol. The factor of 2 is just a matter of convenience. In this case the solution of equation (2.7) reads $[25]$

$$
\gamma_{a}^{k}(A)=\left[1+\theta^{2} A^{2} \chi\left(\theta^{2} A^{2}\right)\right] \delta_{a}^{k}-\theta^{2} \chi\left(\theta^{2} A^{2}\right) A_{a} A^{k}-\varepsilon_{a}{ }^{k l} A_{l},
$$

where

$$
\chi(t)=\frac{1}{t}(\sqrt{t} \cot \sqrt{t}-1), \quad \chi(0)=-\frac{1}{3} .
$$

We use the Kronecker delta to raise and lower indices, and summation under the repeated indices is understood, $A^{2}=A_{m} A^{m}$.

The corresponding non-commutative deformation of Abelian gauge transformations reads [25],

$$
\delta_{f} A_{a}=\partial_{a} f+\left\{A_{a}, f\right\}+\theta \varepsilon_{a}{ }^{k l} A_{k} \partial_{l} f+\theta^{2}\left(\partial_{a} f A^{2}-\partial_{k} f A^{k} A_{a}\right) \chi\left(\theta^{2} A^{2}\right) .
$$

The latter may be verified to close the algebra (2.5) and to reproduce the correct commutative limit $\theta \rightarrow 0$.

This result is essentially different from what one would get in standard approaches. See for example [15], where the infinitesimal transformation of the gauge potential for Lie $\mathfrak{s u}(2)$ type noncommutativity reads $\delta_{f} A_{a}=D_{a} f+i\left[f, A_{a}\right]_{*}$ with $D_{a}=-\frac{i}{\theta}\left[x_{a}, \cdot\right]_{*} \stackrel{\theta \rightarrow 0}{\rightarrow} \epsilon_{a b c} x_{b} \partial_{c}$. Lie algebra type $\star$-commutators do not converge to usual derivations in the commutative limit and the whole gauge theory behaves quite differently from the commutative analogue (see [15] for related duscussion). 


\section{Non-commutative field strength}

In previous section the $\mathrm{U}(1)$ gauge potential has been introduced as a vector-valued element of the NC algebra $\mathcal{A}_{\Theta},\left\{A_{a}\right\}, a=1, \ldots \operatorname{dim} \mathcal{A}_{\Theta}$, whose gauge transformation (2.6) was fixed by the request that it be compatible with the closure of the algebra of gauge parameters (2.5). Similarly, we look here for a deformation of the U(1) field strength,

$$
\mathcal{F}_{a b}=\partial_{a} A_{b}-\partial_{b} A_{a}+\mathcal{O}(\Theta)
$$

which be covariant under gauge transformations (2.6), namely satisfying

$$
\delta_{f} \mathcal{F}_{a b}=\left\{\mathcal{F}_{a b}, f\right\},
$$

where, $\delta_{f} \mathcal{F}_{a b}:=\mathcal{F}_{a b}\left(A+\delta_{f} A\right)-\mathcal{F}_{a b}(A)$. In three space-time dimensions such a field was constructed in [27].

In this section we address the general $n$-dimensional case. Following [27] we are looking for a solution of eq. (3.2) in the form,

$$
\mathcal{F}_{a b}=P_{a b}^{c d}(A) \partial_{c} A_{d}+R_{a b}^{c d}(A)\left\{A_{c}, A_{d}\right\},
$$

where we choose

$$
P_{a b}^{c d}(A)=\delta_{a}^{c} \delta_{b}^{d}-\delta_{a}^{d} \delta_{b}^{c}+\mathcal{O}(\Theta), R_{a b}^{c d}(A)=\frac{1}{2}\left(\delta_{a}^{c} \delta_{b}^{d}-\delta_{a}^{d} \delta_{b}^{c}\right)+\mathcal{O}(\Theta),
$$

to match (3.1). By construction, $R_{a b}{ }^{c d}(A)$ should be antisymmetric in upper indices since it is contracted with the Poisson bracket $\left\{A_{c}, A_{d}\right\}$.

Eq. (3.2), upon replacing the Ansatz (3.3), becomes after simplification,

$$
\begin{array}{r}
{\left[\gamma_{l}^{k} \partial_{A}^{l} P_{a b}{ }^{c d}+\Theta^{k l} \partial_{l} P_{a b}^{c d}+P_{a b}{ }^{c l} \partial_{A}^{d} \gamma_{l}^{k}+P_{a b}^{l d} \partial_{l} \Theta^{c k}+2 R_{a b}^{l d} \partial_{m} \gamma_{l}^{k} \Theta^{m c}\right] \partial_{c} A_{d} \partial_{k} f+} \\
P_{a b}{ }^{c d} \gamma_{d}^{k} \partial_{c} \partial_{k} f+\left[P_{a b}{ }^{c d}-2 \gamma_{l}^{c} R_{a b} l d\right]\left\{A_{d}, \partial_{c} f\right\}+(3.5) \\
{\left[\gamma_{l}^{k} \partial_{A}^{l} R_{a b}{ }^{c d}+\Theta^{k l} \partial_{l} R_{a b}{ }^{c d}+R_{a b}{ }^{c l} \partial_{A}^{d} \gamma_{l}^{k}+R_{a b}^{l d} \partial_{A}^{c} \gamma_{l}^{k}\right]\left\{A_{c}, A_{d}\right\} \partial_{k} f=0 .}
\end{array}
$$

The latter should hold for any gauge parameter $f$ and any gauge field $A_{a}$. Thus, eq. (3.2) yields four separate equations for the coefficient functions $P_{a b}{ }^{c d}$ and $R_{a b}{ }^{c d}$. The first equation involves $P_{a b}{ }^{c d}$ and $R_{a b}{ }^{c d}$,

$$
\gamma_{l}^{k} \partial_{A}^{l} P_{a b}^{c d}+\Theta^{k l} \partial_{l} P_{a b}^{c d}+P_{a b}^{c l} \partial_{A}^{d} \gamma_{l}^{k}+P_{a b}^{l d} \partial_{l} \Theta^{c k}+2 R_{a b}^{l d} \partial_{m} \gamma_{l}^{k} \Theta^{m c}=0 .
$$

The second one,

$$
P_{a b}{ }^{[c d} \gamma_{d}^{k]}=0,
$$

is an algebraic relation on the coefficient $P_{a b} c d$. The third equation relates $P_{a b} c d$ and $R_{a b} c d$,

$$
P_{a b}{ }^{c d}=2 \gamma_{l}^{c} R_{a b}{ }^{l d}
$$

and the last one is an equation for $R_{a b}{ }^{c d}$ reading,

$$
\gamma_{l}^{k} \partial_{A}^{l} R_{a b}^{c d}+\Theta^{k l} \partial_{l} R_{a b}^{c d}+R_{a b}^{c l} \partial_{A}^{d} \gamma_{l}^{k}+R_{a b}^{l d} \partial_{A}^{c} \gamma_{l}^{k}=0 .
$$


To start with, we look for a perturbative solution in $\Theta$ of equation (3.9) using the expression for $\gamma_{l}^{k}$ found previously, eq. (2.8). On using the second of eqs. (3.4) up to first order in $\Theta$ and observing that, at first order $\gamma_{l}^{k(1)}=-\partial_{l} \Theta^{k b} A_{b} / 2$, one obtains from (3.9),

$$
R_{a b}{ }^{c d(1)}=\frac{1}{4}\left(\delta_{a}^{c} \partial_{b} \Theta^{k d}-\delta_{a}^{d} \partial_{b} \Theta^{k c}-\delta_{b}^{c} \partial_{a} \Theta^{k d}+\delta_{b}^{d} \partial_{a} \Theta^{k c}\right) A_{k} .
$$

As for the second order, substituting the latter back into (3.9) and using the expression for $\gamma_{l}^{k(2)}$ given in (2.8) one finds,

$$
\begin{aligned}
R_{a b}{ }^{c d(2)}= & \left(\frac{1}{12} \delta_{a}^{c} \Theta^{n m} \partial_{b} \partial_{m} \Theta^{k d}-\frac{1}{12} \delta_{a}^{d} \Theta^{n m} \partial_{b} \partial_{m} \Theta^{k c}\right. \\
& -\frac{1}{12} \delta_{b}^{c} \Theta^{n m} \partial_{a} \partial_{m} \Theta^{k d}+\frac{1}{12} \delta_{b}^{d} \Theta^{n m} \partial_{a} \partial_{m} \Theta^{k c} \\
& +\frac{1}{12} \delta_{a}^{c} \partial_{b} \Theta^{n m} \partial_{m} \Theta^{k d}-\frac{1}{12} \delta_{a}^{d} \partial_{b} \Theta^{n m} \partial_{m} \Theta^{k c} \\
& -\frac{1}{12} \delta_{b}^{c} \partial_{a} \Theta^{n m} \partial_{m} \Theta^{k d}+\frac{1}{12} \delta_{b}^{d} \partial_{a} \Theta^{n m} \partial_{m} \Theta^{k c} \\
& \left.+\frac{1}{8} \partial_{a} \Theta^{k c} \partial_{b} \Theta^{n d}-\frac{1}{8} \partial_{a} \Theta^{n d} \partial_{b} \Theta^{k c}\right) A_{k} A_{n} .
\end{aligned}
$$

The process can be thus iterated to all orders in $\Theta$.

It is remarkable that since eq. (3.8) expresses the coefficient functions $P_{a b}{ }^{c d}$ in terms of $R_{a b}{ }^{c d}$ and $\gamma_{l}^{c}$, eqs. (3.6) and (3.7) become consistency conditions for the solution of eq. (3.2). One may check that (3.6) holds as a consequence of (3.8), (3.9) and (2.7), while (3.7) is satisfied as a consequence of (3.8) and the antisymmetry of $R_{a b}{ }^{c d}$.

Let us notice that the result we have found for the strength field $\mathcal{F}$ is valid in any dimension, whereas previous result in [27] was specific of three dimensions. Moreover, the latter was only valid for linear $\Theta$, while now we have considered a general dependence in $x$. In the linear case, $\Theta^{k l}(x)=c_{m}^{k l} x^{m}$, the coefficient functions $\gamma_{a}^{k}(A), P_{a b}{ }^{c d}(A)$ and $R_{a b}{ }^{c d}(A)$ are only functions of the gauge field $A$ and do not depend explicitly on coordinates. For general $\Theta^{k l}(x)$ they may have explicit $x$ dependence. This in turn produces additional contributions of the form $\Theta^{k l} \partial_{l} P_{a b} c d, 2 R_{a b}^{l d} \partial_{m} \gamma_{l}^{k} \Theta^{m c}$ and $\Theta^{k l} \partial_{l} R_{a b} c d$ which have been included in eqs. (3.5)-(3.9).

Canonical non-commutativity. Since in this case, $\gamma_{l}^{k}=\delta_{l}^{k}$, one finds from eqs. (3.8), (3.9),

$$
R_{a b}^{c d}(A)=\frac{1}{2}\left(\delta_{a}^{c} \delta_{b}^{d}-\delta_{a}^{d} \delta_{b}^{c}\right), \quad \text { and } \quad P_{a b}{ }^{c d}(A)=\delta_{a}^{c} \delta_{b}^{d}-\delta_{a}^{d} \delta_{b}^{c},
$$

which results in,

$$
\mathcal{F}_{a b}^{c a n}=\partial_{a} A_{b}-\partial_{b} A_{a}+\left\{A_{a}, A_{b}\right\} .
$$

Similarly to the result we found for the gauge potential, if we repeat the procedure just described for a fully canonical non-commutative theory, with Poisson brackets replaced by star commutators, one obtains [24]

$$
\mathcal{F}_{a b}^{N C}=\partial_{a} A_{b}-\partial_{b} A_{a}-i\left[A_{a}, A_{b}\right]_{\star} .
$$

which is consistent with the standard definition of NC field strength (A.8). 
Lie algebra noncommutativity: $\mathbb{R}_{\boldsymbol{\theta}}^{\mathbf{3}}$. For $\mathfrak{s u}(2)$-like noncommutativity, in the slowly varying fields approximation, the matrix $\gamma_{l}^{k}(A)$ was determined in (2.11). The solution of equation (3.9) reads,

$$
\begin{aligned}
R_{a b}{ }^{c d}(A)= & \frac{1}{2}\left(\delta_{a}^{c} \delta_{b}^{d}-\delta_{a}^{d} \delta_{b}^{c}\right) \lambda\left(\theta^{2} A^{2}\right) \\
& +\frac{\theta}{2}\left(\varepsilon_{a b}^{c} A^{d}-\varepsilon_{a b}^{d} A^{c}\right) \lambda\left(\theta^{2} A^{2}\right) \\
& +\frac{\theta^{2}}{2}\left(\delta_{a}^{c} A_{b} A^{d}-\delta_{b}^{c} A_{a} A^{d}-\delta_{a}^{d} A_{b} A^{c}+\delta_{b}^{d} A_{a} A^{c}\right) \lambda^{\prime}\left(\theta^{2} A^{2}\right),
\end{aligned}
$$

where

$$
\lambda(t)=\left(\frac{\sin \sqrt{t}}{\sqrt{t}}\right)^{2}
$$

and $\lambda^{\prime}$ indicates its derivative. The function $\lambda(t)$ satisfies the equation, $\lambda^{\prime}=\chi \lambda$, with initial condition, $\lambda(0)=1$. Being in $3 d$ any totally antisymmetric tensor of rank four vanishes. In particular,

$$
\varepsilon^{a b c} A^{e}-\varepsilon^{b c e} A^{a}+\varepsilon^{c e a} A^{b}-\varepsilon^{e a b} A^{c}=0 .
$$

By taking into account the above relation and its consequences in eq. (3.8) we represent the coefficient $P_{a b}{ }^{c d}$ in a more convenient form,

$$
\begin{aligned}
P_{a b}^{c d}(A)= & \left(\delta_{a}^{c} \delta_{b}^{d}-\delta_{a}^{d} \delta_{b}^{c}\right) \phi\left(\theta^{2} A^{2}\right)+2 \theta \varepsilon_{a b}^{c} A^{d} \phi\left(\theta^{2} A^{2}\right) \\
& -\theta \varepsilon_{a b m} A^{m} \delta^{c d} \lambda\left(\theta^{2} A^{2}\right)-\theta \varepsilon_{a b}{ }^{d} A^{c} \lambda\left(\theta^{2} A^{2}\right) \\
& +\theta^{2}\left(\delta_{a}^{c} A_{b} A^{d}-\delta_{b}^{c} A_{a} A^{d}\right)[\chi \phi-\lambda]\left(\theta^{2} A^{2}\right) \\
& -\theta^{3} \varepsilon_{a b m} A^{m} A^{c} A^{d} \lambda^{\prime}\left(\theta^{2} A^{2}\right)
\end{aligned}
$$

with,

$$
\phi(t)=(1+t \chi(t)) \lambda(t)=\frac{\sin \sqrt{t} \cos \sqrt{t}}{\sqrt{t}} .
$$

It is remarkable that there are only two independent functions $\chi(t)$ and $\lambda(t)$ which determine the whole construction.

In this case we can simplify the expression for the field strength. One may check that,

$$
\partial_{A}^{l} R_{a b}^{c d}+\operatorname{cycl} .(c d l)=0 .
$$

Consequently,

$$
R_{a b}^{c d}=\partial_{A}^{c} \rho_{a b}{ }^{d}-\partial_{A}^{d} \rho_{a b}^{c}
$$

where

$$
\rho_{a b}{ }^{c}=\frac{1}{4}\left(\delta_{b}^{c} A_{a}-\delta_{a}^{c} A_{b}\right) \lambda\left(\theta^{2} A^{2}\right)-\frac{1}{4 \theta} \varepsilon_{a b}^{c} \Lambda\left(\theta^{2} A^{2}\right),
$$

with, $\Lambda^{\prime}(t)=\lambda(t)$. The same procedure can be applied for $P_{a b}{ }^{c d}$. Since,

$$
\partial_{A}^{l} P_{a b}^{c d}=\partial_{A}^{d} P_{a b}^{c l}
$$


we may represent it as,

$$
P_{a b}{ }^{c d}=\partial_{A}^{d} \pi_{a b}{ }^{c}
$$

where

$$
\pi_{a b}^{c}=\left(\delta_{a}^{c} A_{b}-\delta_{b}^{c} A_{a}\right) \phi\left(\theta^{2} A^{2}\right)-\theta \varepsilon_{a b m} A^{m} A^{c} \lambda\left(\theta^{2} A^{2}\right)+\frac{1}{\theta} \varepsilon_{a b}^{c} \Phi\left(\theta^{2} A^{2}\right),
$$

with, $\Phi(t)=\int \phi(t) d t=-\cos (2 \sqrt{t}) / 2$. Then the expression for the non-commutative field strength becomes,

$$
\mathcal{F}_{a b}^{s u(2)}=\partial_{c} \pi_{a b}^{c}+2\left\{\rho_{a b}^{c}, A_{c}\right\} .
$$

In the standard approach the field strength is defined as in (A.3). Then the Bianchi identity is satisfied by definition. See for example [15] for a comparison in case of $\mathfrak{s u}(2)$-like noncommutativity.

Within the present approach Bianchi identity is not automatically built-in because the field strength is not defined as the curvature of a connection. However one may still ask whether the non-commutative field strength (3.26) satisfies some deformed Bianchi identity. We leave it as an open problem.

\section{Noncommutative Chern-Simons model}

Noncommutative deformation of Chern-Simons (CS) theory was constructed in [27]. In this section for completeness and for the convenience of the reader we recollect the main findings of [27]. Just like in the standard commutative case, non-commutative ChernSimons equations are obtained by requiring that the NC Field strength should vanish everywhere. Since we are in three dimensions we may set,

$$
\mathcal{F}^{a}(A):=\frac{1}{2} \varepsilon^{a b c} \mathcal{F}_{b c}=P^{a b c}(A) \partial_{b} A_{c}+R^{a b c}(A)\left\{A_{b}, A_{c}\right\}=0 .
$$

With respect to the comment made at the end of last section, let us notice that this definition of the field strength looks like a deformation of the covariant derivative, through the coefficient functions $P^{a b c}$ and $R^{a b c}$. Eq. (4.1) satisfies the following two requirements. It transforms covariantly under the NC gauge transformations $(2.6), \delta_{f} \mathcal{F}^{a}=\left\{\mathcal{F}^{a}, f\right\}$, and reproduces in the commutative limit, $\Theta \rightarrow 0$, the standard Abelian CS equation of motion, i.e., $\lim _{\Theta \rightarrow 0} \mathcal{F}^{a}(A)=\varepsilon^{a b c} \partial_{b} A_{c}$. These two properties are exactly what we expect from a suitable noncommutative deformation of Chern-Simons theory.

It is important to stress that the noncommutative CS equations (4.1) are nonLagrangian. Indeed, they do not satisfy the criterium of commutation of second variational derivatives,

$$
\frac{\delta \mathcal{F}^{a}}{\delta A^{b}} \neq \frac{\delta \mathcal{F}^{b}}{\delta A^{a}}
$$

This is a main difference between our proposal and previous approaches. It is not based on a deformation of the commutative action as a consequence of a modification of the geometric structures involved, but on the request of gauge covariance and the correct commutative limit of the corresponding field equations. The dynamics that is obtained in such a way may not admit the existence of an action principle, as it is the case for CS equations (4.1). 


\section{Non-commutative Yang-Mills theory}

Having defined the NC field strength as in (3.3), differently form CS dynamics, it is possible for U(1) Yang-Mills theory to introduce a non-commutative deformation by means of an action principle.

On defining the non-commutative Yang-Mills Lagrangian as,

$$
\mathcal{L}=-\frac{1}{4} \mathcal{F}_{a b} \mathcal{F}^{a b}
$$

it is possible to verify that it transforms covariantly under the NC gauge transformations $(2.6), \delta_{f} \mathcal{L}=\{f, \mathcal{L}\}$. Consequently the corresponding action, $S=\int \mathcal{L}$, is gauge invariant, $\delta_{f} S \equiv 0$. By Noether's second theorem the gauge invariance of the action functional implies the existence of non-trivial differential relations (Noether identities) among the corresponding Euler-Lagrange equations. See, e.g., [33] for the derivation of the Noether identities within the $\mathrm{L}_{\infty}$-formalism.

Canonical noncommutativity. For canonical noncommutativity it is particularly easy to derive the equations of motion which yield the noncommutative analogue of Maxwell equations. Taking into account eq. (3.12) for the coefficient functions $R_{a b}{ }^{c d}$ and $P_{a b}{ }^{c d}$ which correspond to constant $\Theta$, we get from the Lagrangian (5.1) Euler Lagrange equations in the form:

$$
\partial_{a} \mathcal{F}_{c a n}^{a d}+\left\{A_{a}, \mathcal{F}_{c a n}^{a d}\right\}=0
$$

where $\mathcal{F}_{a b}^{c a n}$ was found in eq. (3.13) to be

$$
\mathcal{F}_{a b}^{c a n}=\partial_{a} A_{b}-\partial_{b} A_{a}+\left\{A_{a}, A_{b}\right\} .
$$

They correspond to NC Maxwell equations of the standard approach, when the star commutator is replaced with the Poisson bracket, and reproduce the correct commutative limit as expected.

Lie algebra noncommutativity: $\mathbb{R}_{\boldsymbol{\theta}}^{\mathbf{3}}$. Taking into account that for the $\mathfrak{s u}(2)$-like noncommutativity the coefficient functions $R_{a b}{ }^{c d}$ and $P_{a b}{ }^{c d}$ satisfy eqs. (3.20) and (3.23), one finds for the Euler-Lagrange equations corresponding to (5.1),

$$
\mathcal{E}^{d}(A):=\mathcal{D}_{a b}{ }^{d} \mathcal{F}^{a b}=0,
$$

where the field strength is defined in eq. (3.26) and

$$
\mathcal{D}_{a b}{ }^{d} \mathcal{F}^{a b}=\frac{1}{2} P_{a b}{ }^{c d}(A) \partial_{c} \mathcal{F}^{a b}-R_{a b}{ }^{c d}(A)\left\{A_{c}, \mathcal{F}^{a b}\right\} .
$$

By construction these equations are gauge-covariant and reproduce the U(1) Yang-Mills equations in the commutative limit.

It is interesting to notice that eq. (5.4) acquires the form of a deformed covariant derivative, with the coefficient functions $R_{a b}{ }^{c d}$ and $P_{a b}{ }^{c d}$ taking care of the deformation. We plan to come back to this issue elsewhere. 


\section{Conclusions}

In the paper we have proposed a novel approach to U(1) noncommutative gauge theory, which is based on the request that the commutative limit be retrieved for $\Theta \rightarrow 0$ and the dynamics of pure gauge fields be gauge covariant. This is achieved by constructively defining the gauge potential and the field strength through recursive equations which may be solved order by order in the NC parameter, and, generalising previous derivations [24, 25, 27], are valid in any space-time dimension and for generic dependence of $\Theta$ on spacetime coordinates. As for the examples considered, especially interesting is the Lie-algebra type noncommutativity. We are presently investigating another instance of such a family, which is the so-called k-Minkowski spacetime [34].

Two immediate research questions which would be interesting to investigate are the following. First, prior to any quantum field theory application, one should solve the classical equations of motion and check fundamental problems such as the propagation of light in such a deformed space-time. Secondly, one should address the problem of coupling gauge fields with matter fields. Indeed, since the gauge potential is not introduced as a connection one-form, the notion of covariant derivative in such a theory is not automatic. A related problem is the possibility of reformulating the above defined field strength as a consistent deformation of the notion of curvature of the gauge potential. We plan to come back to these issues in the near future.

\section{Acknowledgments}

V.G.K. thanks the University of Naples for hospitality where the present work was initiated.

\section{A Gauge connection}

A natural noncommutative extension of the notion of connection is introduced in $[8,9]$, where one replaces complex vector bundles of physical fields over space-time, with rightmodules, $\mathbb{M}$ over noncommutative space-time, namely the non-commutative algebra $\mathcal{A}$. Generalising the standard definition which is proper of geometric approaches to gauge theory, a connection on $\mathbb{M}$ can be conveniently defined by a linear map $\nabla: \operatorname{Der}(\mathcal{A}) \times \mathbb{M} \rightarrow \mathbb{M}$ satisfying

$$
\nabla_{X}(m f)=m X(f)+\nabla_{X}(m) f, \nabla_{c X}(m)=c \nabla_{X}(m), \nabla_{X+Y}(m)=\nabla_{X}(m)+\nabla_{Y}(m)
$$

for any $X, Y \in \operatorname{Der}(\mathcal{A}), f \in \mathcal{A}, m \in \mathbb{M}, c \in \mathcal{Z}(\mathcal{A})$, the center of the algebra. Hermitian connections satisfy for any real derivation $X \in \operatorname{Der}(\mathcal{A})$

$$
X\left(h\left(m_{1}, m_{2}\right)\right)=h\left(\nabla_{X}\left(m_{1}\right), m_{2}\right)+h\left(m_{1}, \nabla_{X}\left(m_{2}\right)\right), \forall m_{1}, m_{2} \in \mathbb{M},
$$

where $h: \mathbb{M} \otimes \mathbb{M} \rightarrow \mathcal{A}$ denotes a Hermitian structure on $\mathcal{A}$. The curvature is the linear $\operatorname{map} F(X, Y): \mathbb{M} \rightarrow \mathbb{M}$ defined by

$$
F(X, Y) m=\left[\nabla_{X}, \nabla_{Y}\right] m-\nabla_{[X, Y]} m, \forall X, Y \in \operatorname{Der}(\mathcal{A}) .
$$


The group of gauge transformations of $\mathbb{M}, \mathcal{U}(\mathbb{M})$, is defined as the group of automorphisms of $\mathbb{M}$ compatible both with the structure of right $\mathcal{A}$-module and the Hermitian structure, i.e

$$
g(m f)=g(m) f, \quad h\left(g\left(m_{1}\right), g\left(m_{2}\right)\right)=h\left(m_{1}, m_{2}\right) \quad \forall g \in \mathcal{U}(\mathbb{M}), \quad \forall m_{1}, m_{2} \in \mathbb{M}
$$

For any $g \in \mathcal{U}(\mathbb{M})$ we have

$$
\begin{aligned}
\nabla_{X}^{g}: & \mathbb{M} \rightarrow \mathbb{M}, & \nabla_{X}^{g}=g^{-1} \circ \nabla_{X} \circ g \\
F(X, Y)^{g}: & \mathbb{M} \rightarrow \mathbb{M}, & F(X, Y)^{g}=g^{-1} \circ F(X, Y) \circ g .
\end{aligned}
$$

For $\mathrm{U}(1)$ gauge theory, where the relevant vector bundle is a complex line bundle, the corresponding $\mathrm{NC}$ generalisation is a one-dimensional $\mathcal{A}$-module $\mathbb{M}=\mathbb{C} \otimes \mathcal{A}$. As Hermitian structure one chooses $h\left(f_{1}, f_{2}\right)=f_{1}^{\dagger} f_{2}$ and takes real derivations. Then a Hermitian connection is entirely determined by its action on the one-dimensional basis of the module, $\nabla_{X}(\mathbf{1})$. We have $\nabla_{X}(f)=\nabla_{X}(\mathbf{1}) f+X(f)$, with $\nabla_{X}(\mathbf{1})^{\dagger}=\nabla_{X}(\mathbf{1})$. This defines in turn the gauge connection 1 -form, $A$, by means of

$$
A: X \rightarrow A(X):=i \nabla_{X}(\mathbf{1}), \quad \forall X \in \operatorname{Der}(\mathcal{A})
$$

From the compatibility condition with the Hermitian structure, eq. (A.4), one obtains that gauge transformations are the group of unitary elements of the noncommutative algebra $\mathcal{A}$. Indeed, on using $g(f)=g(\mathbf{1} f)=g(\mathbf{1}) \star f$ and imposing compatibility, one obtains $h\left(g\left(f_{1}\right), g\left(f_{2}\right)\right)=h\left(f_{1}, f_{2}\right)$ which implies $g(\mathbf{1})^{\dagger} \star g(\mathbf{1})=\mathbf{1}$. We pose $g(\mathbf{1}) \equiv g \in \mathcal{U}(\mathcal{A})$ the group of unitary elements of the NC algebra $\mathcal{A}$, acting multiplicatively on $\mathcal{A}$ from the left.

To give an explicit example of the whole construction, let us consider the 2-dimensional Moyal plane, $\mathcal{A}=\mathbb{R}_{\theta}^{2}$, with constant noncommutative parameter, $\theta$. The latter is referred to as canonical noncommutativity in the paper. The algebra of derivations is in this case the Abelian algebra generated by derivatives $\partial_{\mu}$. From eqs. (A.5), (A.6) we obtain

$$
F_{\mu \nu}=F\left(\partial_{\mu}, \partial_{\nu}\right)=\partial_{\mu} A_{\nu}-\partial_{\nu} A_{\mu}-i\left[A_{\mu}, A_{\nu}\right]_{\star}
$$

with $A_{\mu}=i \nabla_{\mu}(\mathbf{1})$, and, to make contact with usual notation, we have rescaled $F$ by a factor of $i$. The unitary gauge group $\mathcal{U}\left(\mathbb{R}_{\theta}^{2}\right)$ acts as $\nabla_{\mu}^{g}=g^{\dagger} \circ \nabla_{\mu} \circ g$, yielding

$$
A_{\mu}^{g}=g \star A_{\mu} \star g^{\dagger}-i \partial_{\mu} g \star g^{\dagger}, F_{\mu \nu}^{g}=g \star F_{\mu \nu} \star g^{\dagger}, \quad \forall g \in \mathcal{U}(\mathcal{A})
$$

Being unitary elements of $\mathcal{A}$ gauge transformations may be written as star exponentials

$$
g[f]=\exp _{\star}(i f)
$$

and the star exponential is by definition

$$
\exp _{\star}(i f) \equiv \sum_{n=0}^{\infty} \frac{(i)^{n}}{n !} \underbrace{f \star \ldots \star f}_{n \text { times }}
$$

where the gauge parameters $f$ are functions of $x \in \mathbb{R}_{\theta}^{2}$. Hence, for the Moyal plane we get infinitesimal gauge transformations in the form

$$
\delta A_{\mu}=\partial_{\mu} f+i\left[f, A_{\mu}\right]_{\star} \quad \delta F_{\mu \nu}=i\left[f, F_{\mu \nu}\right]_{\star} .
$$


$\mathrm{U}(N)$ gauge theory is generalised to the $\mathrm{NC}$ case along the same lines. Matter fields are represented by complex $\mathcal{A}$ modules $\mathbb{M}=\mathbb{C}^{N} \otimes \mathcal{A}$ while gauge transformations are automorphisms of $\mathbb{M}$ which may be realised as Lie algebra valued $\star$-exponentials according to

$$
g(f)=\exp _{\star}\left(i f^{j} e_{j}\right), \quad j=1, \ldots, N
$$

with $f^{j} \in \mathcal{A}$ and $e_{j}$ a Hermitian basis in $\mathbb{C}^{N}$.

Open Access. This article is distributed under the terms of the Creative Commons Attribution License (CC-BY 4.0), which permits any use, distribution and reproduction in any medium, provided the original author(s) and source are credited.

\section{References}

[1] S. Doplicher, K. Fredenhagen and J.E. Roberts, The quantum structure of space-time at the Planck scale and quantum fields, Commun. Math. Phys. 172 (1995) 187 [hep-th/0303037] [INSPIRE].

[2] S. Minwalla, M. Van Raamsdonk and N. Seiberg, Noncommutative perturbative dynamics, JHEP 02 (2000) 020 [hep-th/9912072] [INSPIRE].

[3] A. Matusis, L. Susskind and N. Toumbas, The IR/UV connection in the noncommutative gauge theories, JHEP 12 (2000) 002 [hep-th/0002075] [INSPIRE].

[4] I.E. Segal, Quantized differential forms, Topology 7 (1968) 147.

[5] I.E. Segal, Quantization of the de Rham complex, Proc. Sympos. Pure Math. 16 (1970) 205.

[6] G. Landi and G. Marmo, Algebraic differential calculus for gauge theories, Nucl. Phys. B Proc. Suppl. 18A (1990) 171 [InSPIRE].

[7] J. Wess, Deformed coordinate spaces: derivatives, in Proceeedings of the $1^{\text {st }}$ Balkan workshop on mathematical, theoretical and phenomenological challenges beyond the Standard Model: perspectives of Balkans collaboration, World Scientific, Singapore (2003), pg. 122 [hep-th/0408080] [INSPIRE].

[8] M. Dubois-Violette, Dérivations et calcul différentiel non commutatif (in French), Compt. Rend. Acad. Sci. Ser. I Math. 307 (1988) 403.

[9] M. Dubois-Violette and P.W. Michor, Dérivations et calcul différentiel non commutatif. II (in French), Compt. Rend. Acad. Sci. Ser. I Math. 319 (1994) 927 [hep-th/9406166] [INSPIRE].

[10] J.-C. Wallet, Derivations of the Moyal algebra and noncommutative gauge theories, SIGMA 5 (2009) 013.

[11] E. Cagnache, T. Masson and J.-C. Wallet, Noncommutative Yang-Mills-Higgs actions from derivation-based differential calculus, J. Noncommut. Geom. 5 (2011) 39 [arXiv:0804.3061] [INSPIRE].

[12] J. Madore, S. Schraml, P. Schupp and J. Wess, Gauge theory on noncommutative spaces, Eur. Phys. J. C 16 (2000) 161 [hep-th/0001203] [INSPIRE].

[13] P. Vitale and J.-C. Wallet, Noncommutative field theories on $R_{\lambda}^{3}$ : toward UV/IR mixing freedom, JHEP 04 (2013) 115 [Addendum ibid. 03 (2015) 115] [arXiv:1212.5131] [INSPIRE].

[14] P. Vitale, Noncommutative field theory on $R_{\lambda}^{3}$, Fortsch. Phys. 62 (2014) 825 [arXiv: 1406.1372] [INSPIRE]. 
[15] A. Géré, P. Vitale and J.-C. Wallet, Quantum gauge theories on noncommutative three-dimensional space, Phys. Rev. D 90 (2014) 045019 [arXiv: 1312.6145] [InSPIRE].

[16] G. Marmo, P. Vitale and A. Zampini, Noncommutative differential calculus for Moyal subalgebras, J. Geom. Phys. 56 (2006) 611 [hep-th/0411223] [INSPIRE].

[17] G. Marmo, P. Vitale and A. Zampini, Derivation based differential calculi for noncommutative algebras deforming a class of three dimensional spaces, J. Geom. Phys. 136 (2019) 104 [arXiv: 1805.06300] [INSPIRE].

[18] D.V. Vassilevich, Twist to close, Mod. Phys. Lett. A 21 (2006) 1279 [hep-th/0602185] [INSPIRE].

[19] M. Chaichian, A. Tureanu and G. Zet, Twist as a symmetry principle and the noncommutative gauge theory formulation, Phys. Lett. B 651 (2007) 319 [hep-th/0607179] [INSPIRE].

[20] M. Chaichian and A. Tureanu, Twist symmetry and gauge invariance, Phys. Lett. B 637 (2006) 199 [hep-th/0604025] [INSPIRE].

[21] P. Aschieri, M. Dimitrijević, F. Meyer, S. Schraml and J. Wess, Twisted gauge theories, Lett. Math. Phys. 78 (2006) 61 [hep-th/0603024] [INSPIRE].

[22] P. Aschieri, F. Lizzi and P. Vitale, Twisting all the way: from classical mechanics to quantum fields, Phys. Rev. D 77 (2008) 025037 [arXiv:0708.3002] [InSPIRE].

[23] M. Kontsevich, Deformation quantization of Poisson manifolds. 1, Lett. Math. Phys. 66 (2003) 157 [q-alg/9709040] [InSPIRE].

[24] R. Blumenhagen, I. Brunner, V. Kupriyanov and D. Lüst, Bootstrapping non-commutative gauge theories from $L_{\infty}$ algebras, JHEP 05 (2018) 097 [arXiv: 1803.00732] [INSPIRE].

[25] V.G. Kupriyanov, $L_{\infty}$-bootstrap approach to non-commutative gauge theories, Fortsch. Phys. 67 (2019) 1910010 [arXiv: 1903.02867] [INSPIRE].

[26] O. Hohm and B. Zwiebach, $L_{\infty}$ algebras and field theory, Fortsch. Phys. 65 (2017) 1700014 [arXiv: 1701.08824] [INSPIRE].

[27] V.G. Kupriyanov, Non-commutative deformation of Chern-Simons theory, Eur. Phys. J. C 80 (2020) 42 [arXiv: 1905.08753] [INSPIRE].

[28] A.B. Hammou, M. Lagraa and M.M. Sheikh-Jabbari, Coherent state induced star product on $R_{\lambda}^{3}$ and the fuzzy sphere, Phys. Rev. D 66 (2002) 025025 [hep-th/0110291] [INSPIRE].

[29] J.M. Gracia-Bondia, F. Lizzi, G. Marmo and P. Vitale, Infinitely many star products to play with, JHEP 04 (2002) 026 [hep-th/0112092] [INSPIRE].

[30] V. Gáliková, S. Kováčik and P. Prešnajder, Laplace-Runge-Lenz vector in quantum mechanics in noncommutative space, J. Math. Phys. 54 (2013) 122106 [arXiv:1309.4614] [INSPIRE].

[31] V.G. Kupriyanov, A hydrogen atom on curved noncommutative space, J. Phys. A 46 (2013) 245303 [arXiv: 1209.6105] [INSPIRE].

[32] V.G. Kupriyanov and P. Vitale, Noncommutative $R^{d}$ via closed star product, JHEP 08 (2015) 024 [arXiv: 1502.06544] [INSPIRE].

[33] M.D. Ćirić, G. Giotopoulos, V. Radovanović and R.J. Szabo, $L_{\infty}$-algebras of Einstein-Cartan-Palatini gravity, arXiv:2003.06173 [INSPIRE].

[34] V. Kupriyanov, M. Kurkov and P. Vitale, A novel approach to gauge theory on k-Minkowski space-time, in preparartion. 